\title{
Testing Efficacy in Determination of Recurrent Supraventricular Tachycardia among Subjectively Symptomatic Children Following "Successful” Ablation
}

\author{
DEEPTI P. BHAT, M.D., WEI DU, PH.D., and PETER P. KARPAWICH, M.Sc., M.D. \\ From the Section of Pediatric Cardiology, Carmen and Ann Adams Department of Pediatrics, The Children's \\ Hospital of Michigan, Wayne State University School of Medicine, Detroit, Michigan
}

Background: Supraventricular tachycardia (SVT) in children can be successfully terminated using catheter ablation techniques; however, chronic, true success may be less. Sensed tachycardia (ST) is common following ablation and the differentiation from true arrhythmia recurrences can be challenging.

Methods: Records of all patients $\leq 18$ years who underwent successful ablation for simple forms of SVT and followed-up for at least a year between 2002 and 2012 were reviewed. Patients with congenital heart disease, atrial muscle tachycardia, and associated ventricular tachycardia were excluded. The diagnostic utility of commonly performed tests was assessed.

Results: Among the 205 eligible subjects, 202 underwent successful ablation (98.5\%), of who five were lost to follow-up before 1 year. The early success rate ( 6 weeks postablation) and mid-term success rate (1 year postablation) were high ( $97.5 \%$ and $87.4 \%$, respectively) whereas the chronic success (5 years postablation) was only $75 \%$. Although true arrhythmia recurrence was significantly higher in the young (mean 11.5 years vs 13.5 years, $P=0.03)$ and males $(P=0.02)$, the presence of diffuse, right-sided fibers was the only independent predictor of true recurrence (odds ratio $=2.7, P=0.03,95 \%$ confidence interval 1.1, 6.8). Significant ST was reported by 111 patients (56\%). The 30-day ambulatory event monitor had the highest sensitivity (71\%) when compared to exercise test (19\%) and electrocardiogram (24\%) in identifying true arrhythmia recurrence.

Conclusions: Acute and early success may not guarantee chronic ablation success. Postablation, symptomatic patients can be most effectively evaluated using ambulatory event monitoring; however, true recurrence may still be missed and requires closer monitoring. (PACE 2014; 37:1009-1016)

supraventricular tachycardia, recurrence, sensed tachycardia, diagnostic test

\section{Introduction}

Supraventricular tachycardia (SVT) is the most common symptomatic arrhythmia in childhood and can be a recurrent and persistent condition. The incidence of this condition is estimated to be one in 100 for children of all ages and one in 200 to 250 for neonates. ${ }^{1,2}$ Most forms of reentrant SVTs involve an accessory conduction pathway that participates in initiation and propagation of tachycardia and is amenable to ablation. While medical therapy is effective in reducing frequency

Disclosures: None.

Conflict of Interest: None.

Address for reprints: Peter P. Karpawich, M.Sc., M.D., Cardiac Electrophysiology, Pediatric Cardiology, The Children's Hospital of Michigan, 3901 Beaubien Blvd, Detroit, MI 48201. Fax: 313-993-0894; e-mail: pkarpawi@dmc.org

Received September 4, 2013; revised January 13, 2014; accepted February 5, 2014.

doi: 10.1111/pace.12373 of episodes in some cases, ablation of the accessory pathways (APs) in the electrophysiology (EP) laboratory, using cryotherapy or radiofrequency energies, remains the treatment of choice for symptomatic older children. ${ }^{3-5}$ Several studies have shown extremely high rates of acute success with ablation $(>96-98 \%))^{5,6}$ However, data are limited on long-term recurrence rates of SVT, and therefore true success, in this population. ${ }^{7}$

It is recognized that from $30 \%$ to $60 \%$ of patients continue to experience sensed tachycardia (ST) in the postablation period., ${ }^{4,8}$ All episodes of ST, however, do not indicate true arrhythmia recurrence and may represent heightened sensitivity to sinus tachycardia. These patients are routinely evaluated by means of several clinical tests including resting electrocardiogram (ECG), exercise stress testing, and ambulatory event monitoring (AEM). While these tests are useful in the initial diagnosis of SVT, their diagnostic yield in predicting arrhythmia recurrence in the postablation period has not been studied. ${ }^{9-13}$

(C)2014 Wiley Periodicals, Inc. 
This study was designed to assess, first, the incidence of ST in children who have undergone successful SVT ablation and, second, to evaluate the diagnostic utility of various clinical tests in identifying true arrhythmia recurrence.

\section{Methods}

This was a retrospective study conducted at The Children's Hospital of Michigan. The study was approved by the Institutional Review Board, Wayne State University School of Medicine. All patients up to 18 years of age who were diagnosed with any form of SVT and subsequently had undergone radiofrequency or cryocatheter ablation in our EP laboratory between 2002 and 2012 were identified. Those patients who had successful ablation and were followed in our clinic for a minimum duration of 1 year were included. Patients with atrial muscle tachycardia, structural congenital heart defects (CHD), those with associated ventricular tachycardia, and with a history of a surgical maze procedure for SVT were excluded. Also, to facilitate correlation of complaints with results, only those patients who underwent ablation for typical forms of reentrant SVT as the primary tachycardia substrate were included.

All clinical, intracardiac electrophysiological study (EPS), and follow-up data on the study group were reviewed. This included demographic data, type of SVT, findings during EPS, follow-up data after the initial ablation including symptoms, results of investigations for evaluation of ST, and any documented episodes of recurrent SVT.

\section{Ablation Procedure}

All patients underwent established EPS under the same protocol by two pediatric electrophysiologists. All procedures were performed under standard general anesthesia. A three-dimensional (3D) mapping system (St. Jude Medical, Minneapolis, MN, USA) was used to define anatomical and electrical landmarks for all patients post mid-2007. Standard programmed electrical stimulation (PES) protocols were instituted to induce tachycardia including risk stratification of each patient with evaluation of refractory periods and inducibility of arrhythmias. Once the arrhythmia substrate and any APs were identified, radiofrequency ablation (RFA) and/or cryoablations were performed per protocol. All patients were observed for 60 minutes following apparently successful ablation. This was followed by a full repeat PES with and without isoproterenol to evaluate ability to reinduce any arrhythmia, as well as administration of adenosine to confirm evidence of any residual accessory orthodromic connections. All patients were observed overnight and evaluated by means of a standard 15-lead ECG to reconfirm a normal
QRS and echocardiogram/Doppler to evaluate atrioventricular valve integrity and effusion.

\section{Follow-Up after Ablation}

All patients were advised to have a follow-up visit at 1 week and between 4 weeks and 6 weeks following ablation in our EP clinic, and then routinely at 1 year. Subsequent follow-up was advised as needed for any symptoms. At the first postablation visit, all patients were evaluated with a detailed history and standard 15-lead ECG. An exercise stress test (ET) depending on the patient's ability to perform the test, as well as history and ECG, were scheduled at the 4-6-week visit. If patients complained of any ST during any of the subsequent follow-up visits, they were additionally evaluated by means of an ECG, AEM, and/or ET as per clinician's decision. If patients presented to the Emergency Room with ST and had documented arrhythmia recurrence, medical management was initiated. A repeat EP procedure was performed based on clinical presentation of the patient.

\section{Statistics}

All data were reported as mean and standard deviation for continuous variables and frequency for categorical variables. For statistical analysis, patients who presented with ST following the initial ablation were selected. Kaplan-Meier curves were constructed to analyze survival free of true recurrence following ablation. Patients with and without a true recurrence were compared using independent sample $t$-test, Mann-Whitney U test, Fisher's exact, or $\chi^{2}$ tests depending on the distribution of the data. Mutivariate logistic regression was performed using recurrence as dependent variable and adjusted odds' ratio with 95\% confidence interval was calculated. The results of various diagnostic modalities done to evaluate ST were analyzed. Sensitivity, specificity, and diagnostic yield of each modality were calculated in identifying true arrhythmia recurrence. All statistical analyses were performed using SPSS version 18.0 (IBM Corp., Armonk, NY, USA).

\section{Results}

During the 10-year study period, a total of 515 patients underwent ablation procedures in our EP lab. Of these, 197 patients met the eligibility criteria for inclusion in this study. The mean age at ablation was $12.7 \pm 3.9$ years and the majority of the patients were males $(\mathrm{N}=$ $120,61 \%)$. Patients were followed for a mean duration of $71 \pm 40$ months (median 66 months). Figure 1 illustrates the Kaplan-Meier curve for freedom from arrhythmia recurrences. There was no significant difference between the duration 


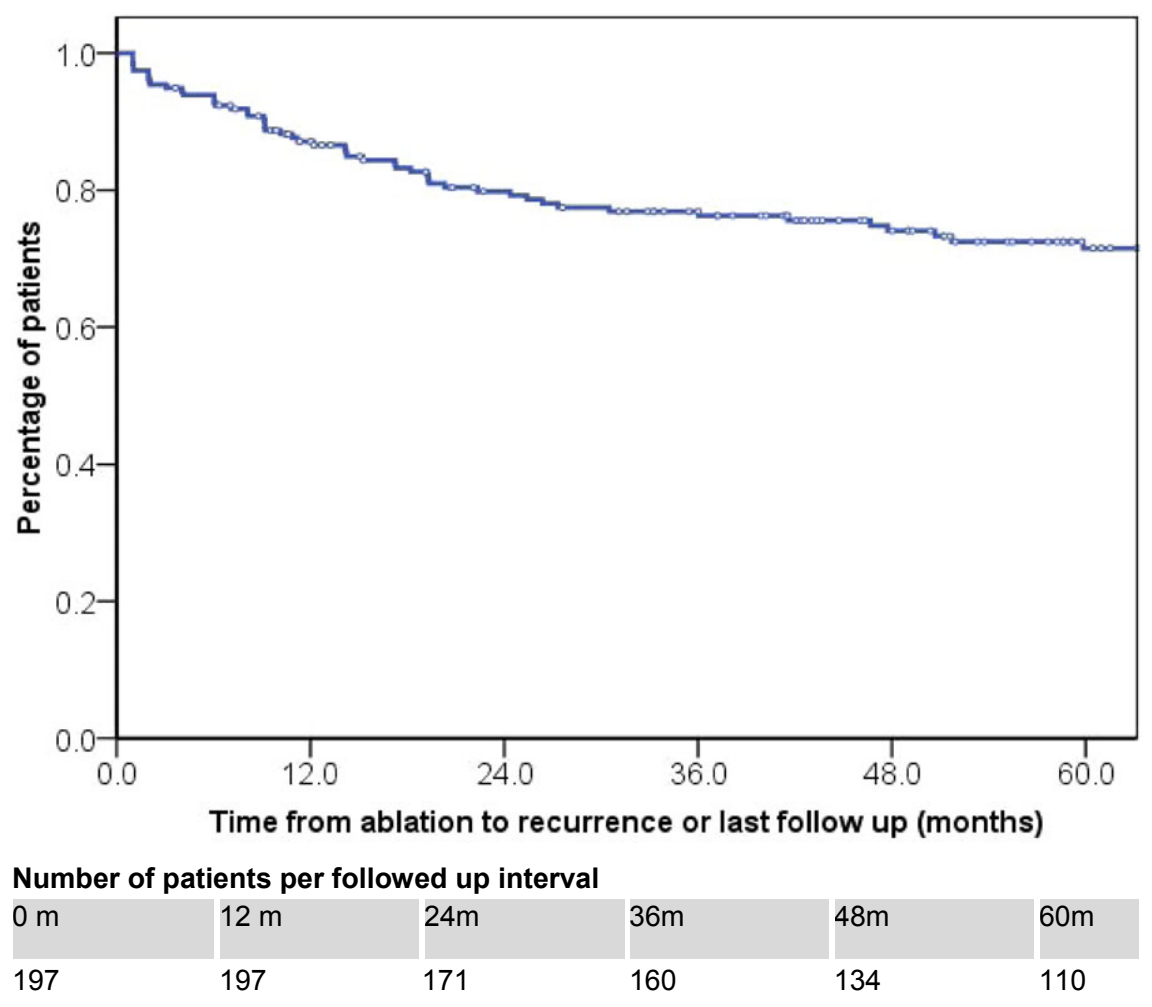

Figure 1. Kaplan-Meier curve for freedom from arrhythmia recurrences.

of follow-up among patients with and those without recurrence. Patients with true arrhythmia recurrence were significantly younger at the time of initial ablation and were more likely to be males (Table I). Figure 2 provides patient numbers during follow-up separated by "success" category: acute, early, mid-term, and chronic, as described below.

\section{Acute Success Rate}

Following ablation, all patients were observed for 60 minutes in the EP lab. Acute success was defined as: (1) a normal QRS and no visible preexcitation among those patients with previous preexcitation, (2) inability to reinduce arrhythmia in spite of repeat PES with and without isoproterenol infusion, (3) no evidence of residual preexcitation following adenosine administration. Three patients continued to have preexcitation following ablation (acute success rate: $98.5 \%$ ).

\section{Early Success Rate}

Early success rate was defined as freedom from clinical arrhythmia recurrence at the time of first follow-up visit at 4-6 weeks and was achieved in $97.5 \%$ patients. Five patients $(2.5 \%)$ had clinical arrhythmia recurrence. None of these patients had residual preexcitation or any abnormal rhythm on resting ECG.

\section{Mid-Term Success Rate}

Mid-term success was defined as freedom from arrhythmia recurrence at 1 year and was $87.4 \%$. Of the 20 patients who had clinical recurrence between 6 weeks and 1 year, six (30\%) had residual preexcitation on their follow-up ECG and one $(5 \%)$ had persistent preexcitation during ET.

\section{Chronic Success Rate}

Chronic persistent success was defined as freedom from clinical arrhythmia recurrence at 5 years postsuccessful ablation and was about $75 \%$. Fifty patients $(25 \%)$ had a clinical arrhythmia recurrence by 5 years, of who $25(12.5 \%)$ had recurrence after 1-year postablation. Of these 25 patients, six (24\%) had residual preexcitation on routine ECG and two had persistent preexcitation during ET.

\section{Arrhythmia Substrate in Patients with True Arrhythmia Recurrence}

During the initial ablation, RFA and/or cryo were used depending on the location of the APs. Cryoablation with or without RFA was 
Table I.

Comparison of Patients with and Without Clinical Arrhythmia Recurrence Following Successful Tachycardia Ablation $(\mathrm{N}=$ 197)

\begin{tabular}{|c|c|c|c|c|}
\hline & $\begin{array}{l}\text { Recurrence } \\
\quad(\mathrm{N}=50)\end{array}$ & $\begin{array}{l}\text { No Recurrence } \\
\quad(\mathrm{N}=147)\end{array}$ & $\begin{array}{c}P \\
\text { Value }\end{array}$ & $\begin{array}{l}\text { OR }(95 \% \mathrm{Cl}) \\
\text { Adjusted Pt }\end{array}$ \\
\hline Age at ablation (years) & $11.7 \pm 4.2$ & $13.5 \pm 3.4$ & 0.003 & NS \\
\hline Gender (male) & $34(68 \%)$ & $36(24 \%)$ & 0.001 & NS \\
\hline \multicolumn{5}{|l|}{ Type of tachycardia } \\
\hline \multicolumn{5}{|c|}{ Atrioventricular reciprocating tachycardia } \\
\hline Right-sided fibers & $25(50 \%)$ & $48(33 \%)$ & 0.04 & \\
\hline discrete AP $\ddagger$ & $12(24 \%)$ & $28(19 \%)$ & NS & \\
\hline diffuse nondiscrete AP & $13(26 \%)$ & $20(14 \%)$ & 0.04 & $2.7(1.1,6.8), 0.03 \dagger$ \\
\hline Left-sided AP & $6(12 \%)$ & $51(35 \%)$ & 0.02 & NS \\
\hline Bilateral AP & $4(8 \%)$ & $5(3 \%)$ & NS & \\
\hline Atrioventricular nodal tachycardia & $13(27 \%)$ & $37(25 \%)$ & NS & \\
\hline Ectopic atrial tachycardia & $2(4 \%)$ & $4(3 \%)$ & NS & \\
\hline Sinus node reentry tachycardia & $0(0 \%)$ & $2(1 \%)$ & NS & \\
\hline $\begin{array}{l}\text { Duration of follow-up (months) } \\
\text { Mean } \pm \text { SD }\end{array}$ & $79.8 \pm 38.2$ & $68.3 \pm 41.3$ & NS & \\
\hline Median (interquartile range) & $76(50,103)$ & $61(40,94)$ & & \\
\hline
\end{tabular}

${ }^{\dagger}$ Multivariate logistic regression analysis. $\mathrm{AP}=$ accessory pathway; $\mathrm{Cl}=$ confidence interval; $\mathrm{OR}=$ odds ratio; $\mathrm{SD}=\mathrm{standard}$ deviation.

preferably used for atrioventricular node reentrant tachycardia (AVNRT) and right anteroseptal fibers to optimize avoidance of atrioventricular node conduction damage. RFA alone was preferred for left-sided fibers. Electroanatomic 3D mapping was used for all patients post mid-2007. The incidence of right-sided fibers was significantly higher in patients with true recurrence (Table I). On multivariate regression analysis, the presence of APs in a right-sided diffuse distribution pattern at initial ablation was the only independent risk factor for recurrence. Forty-six patients underwent repeat EP study for management of arrhythmia recurrence. Twenty-one patients $(46 \%)$ had a residual arrhythmia substrate/AP location similar to the initial EP ablation. Thirteen patients (28\%) had a new substrate/AP in a different location. Eight patients $(17 \%)$ were found to have AP in a new location in addition to the residual AP from initial ablation. Atrial flutter was induced in two patients and no arrhythmia could be induced in two patients $(4 \%)$.

\section{Evaluation of ST}

Of the 197 patients, 111 patients (56\%) reported significant ST which was defined as recurrent, severe subjective sensation of palpitations interfering with patient's daily activities and/or requiring emergency room visit over a 5-year follow-up period after initial ablation (Table II). These patients were evaluated with ECG, ET, and
AEM. Patients received ambulatory monitors typically based on age and potential for compliance. Younger patients typically received "nonlooping" monitors, as compliance with wearing a monitor continuously is often less-than-optimal. The AEM was the most sensitive and specific test with a high negative predictive value (Fig. 3). ECG and ET were found to have limited utility in predicting true arrhythmia recurrence.

\section{Discussion}

SVT is the most common arrhythmia in childhood and typically is associated with accessory bypass pathways for propagation. The destruction or modification of these pathways using ablation therapy is a safe and effective mode of treatment for older children. While several authors have reported extremely high acute success rates of eliminating the tachycardia with ablation, the long-term prognosis for these patients has not been adequately studied. Our study aims to fill this gap by investigating the long-term success rates in a growing pediatric population following ablation for SVT. The results of acute success rates following ablation were comparable with previous studies, approaching nearly $100 \%$. However, over an average followup duration of 6 years, more than half of the patients reported repeated subjective sensations of tachycardia. About one-third of these patients 


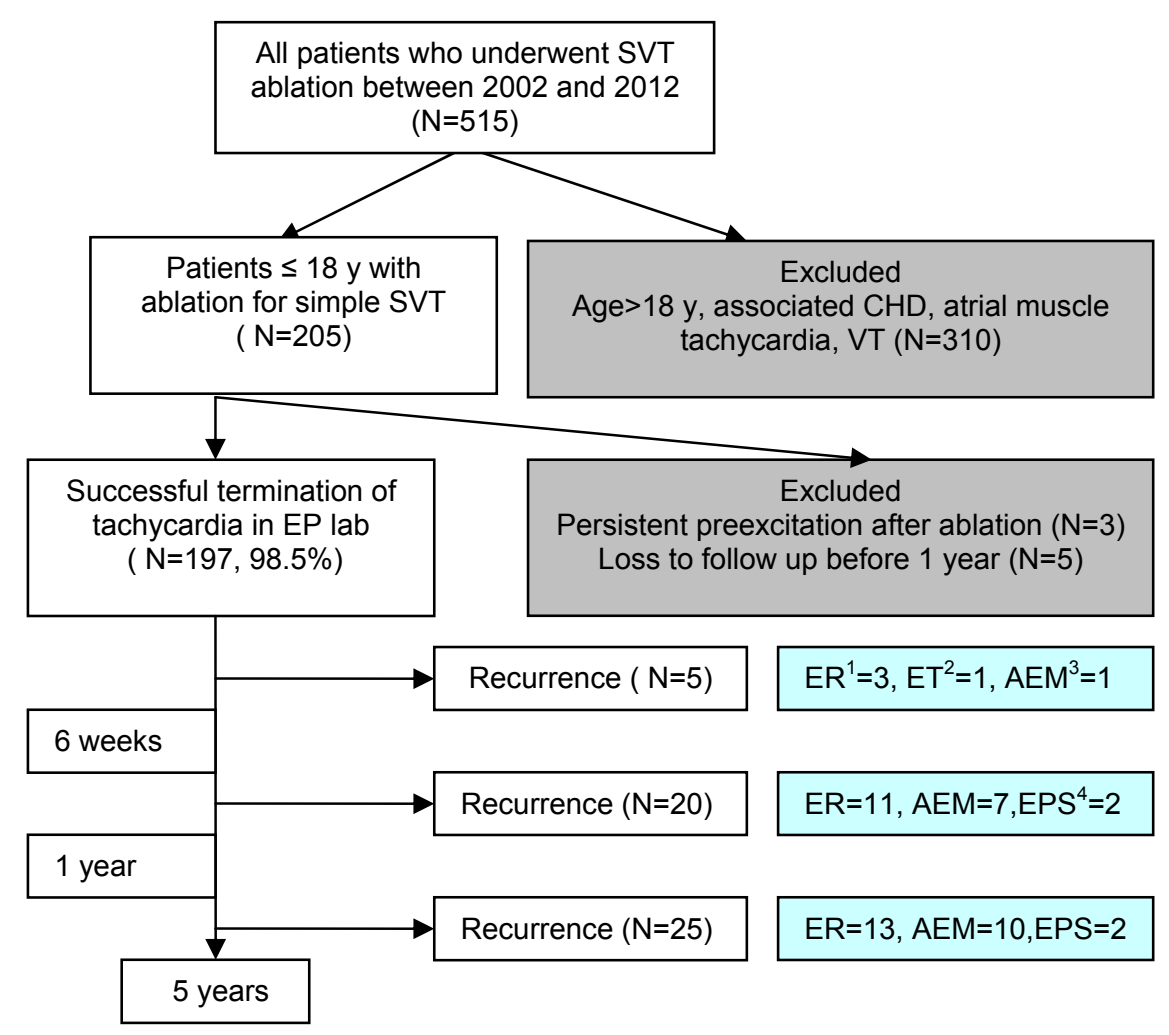

Mode of diagnosis: 1. Emergency room visit 2. Exercise test 3. Ambulatory event monitor 4. Electrophysiology study

Figure 2. Summary of study design and results. $C H D=$ congenital heart disease; EP = electrophysiology; $S V T=$ supraventricular tachycardia; $V T=$ ventricular tachycardia .

had true documented recurrence of the SVT. These results therefore indicate that acute success in eliminating tachycardia may not always translate into long term, true success. This is also the first study that investigates the relative utility of clinical testing in identifying true arrhythmia recurrence among the patients presenting with significant ST following ablation.

Some authors have previously reported postablation outcomes. LaPage et al. studied 61 pediatric patients who underwent cryoablation for AVNRT. ${ }^{3}$ About $15 \%$ patients had recurrent SVT over 2 years. Our current report presents data on a much larger group over a longer follow-up period with a recurrence rate of $25 \%$. Unlike the previous study, our sample was not limited to AVNRT or cryoablation. Chetaille et al. studied outcomes of RFA in 83 patients with CHD over a mean followup duration of $44 \pm 35$ months with a chronic success rate of $59 \%$ of procedures. ${ }^{14}$ In our current study, the follow-up duration was $71 \pm 40$ months, the longest reported postablation interval in pediatric population to date. We excluded patients with CHD due to heterogeneity of this population and the inherent differences in their conduction system when compared to the children with structurally normal heart. ${ }^{15}$ In this aspect, we believe this report more accurately reflects true ablation success in the real world of pediatric tachycardia management, ablation, and growing children.

Our study shows that the location of the AP was the only independent predictor of arrhythmia recurrence a 5 years. Patients with diffuse right-sided fibers at initial ablation were three times more likely to have arrhythmia recurrence compared to those with discrete fibers. In a previous study, Buddhe et al. compared ablation energy sources (RFA vs cryoablation) with recurrences among 87 pediatric patients. ${ }^{5}$ The overall recurrence rate was similar in two groups ( $22 \%$ vs $20 \%$, respectively) over 5 years, with location as an important variable for recurrences. That study showed a predominance of right-sided fibers similar to our current study. Van Hare 


\section{Table II.}

Evaluation of Significant Sensed Tachycardia in Patients with and without Recurrence at 5 Years Postablation (N = 111)

\begin{tabular}{lcc}
\hline & $\begin{array}{c}\text { Recurrence } \\
(\mathbf{N}=\mathbf{5 0 )}\end{array}$ & $\begin{array}{c}\text { No Recurrence } \\
(\mathbf{N}=\mathbf{6 1 )}\end{array}$ \\
\hline Electrocardiogram† & $12 / 50(24 \%)$ & $4 / 61(6 \%)$ \\
Exercise stress testł & $5 / 26(19 \%)$ & $0 / 20(0 \%)$ \\
Ambulatory event monitor & $18 / 25(72 \%)$ & $0 / 38(0 \%)$ \\
$\quad$ Looping recorder & $10 / 14(71 \%)$ & $0 / 18(0 \%)$ \\
Non looping recorder & $8 / 10(80 \%)$ & $0 / 17(0 \%)$ \\
ECAT/Implantable loop recorders & $1 / 2(50 \%)$ & $0 / 0(0 \%)$ \\
\hline
\end{tabular}

${ }^{\dagger}$ Abnormal test defined as persistent preexcitation at rest, ${ }^{\ddagger}$ abnormal test defined as persistent preexcitation and/or documented SVT with exercise.

ECAT $=$ extracardiac ambulatory telemetry.

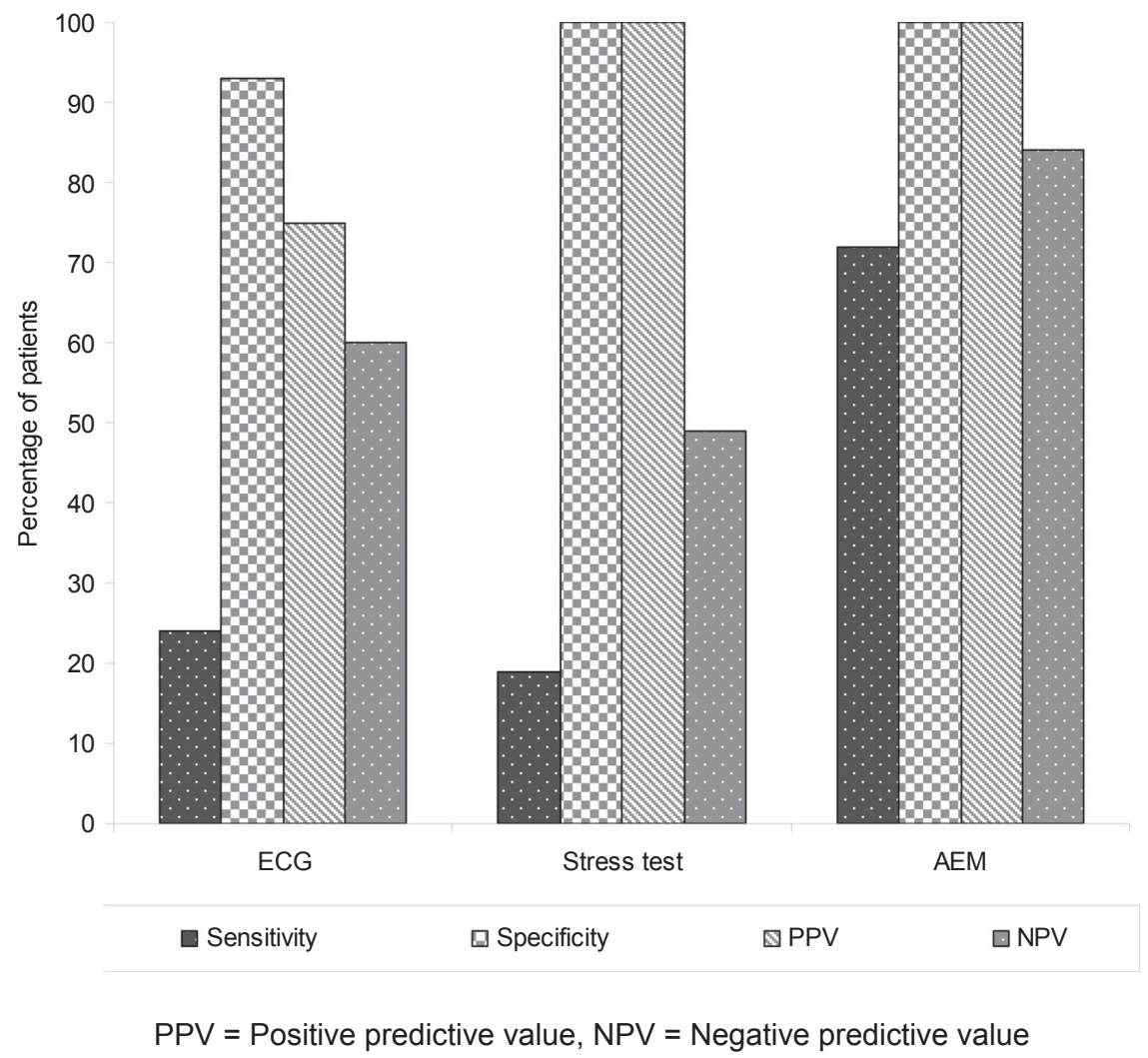

Figure 3. Diagnostic utility of tests performed to evaluate sensed tachycardia $(N=111)$.

et al. conducted a large prospective study of 2,761 pediatric patients to evaluate outcomes after SVT ablation. ${ }^{6}$ The acute success rate for left-sided APs (98\%) was significantly higher than the right-sided fibers $(91 \%)$. The study however did not assess long-term outcomes.

The reasons for increased recurrence with diffuse right-sided fibers are purported to be associated with anatomy. Diffuse arrangements of fibers are identified during mapping/ablation when the ablation catheter crosses at least two "adjacent" mapping regions (e.g., anterior/anteroseptal). ${ }^{16}$ Of course, it is well known that these "regions" are hypothetical and based more on fluorscopic/3D mapping appearances than any actual anatomical references. These diffuse fibers are characterized histologically by wide AP branching and spatial separation and may present with a clinical 
picture of multiple or broad-distribution connections, hindering effective catheter mapping and ablation. ${ }^{17,18}$ The associated folding of the atrium over the ventricle, inherent anatomical variability of the right-sided AV ring, AP physical characteristics, and orientation relative to atrial and ventricular myocardium may all contribute to an inherent overlap among the sites, especially on the right. In the previous era of surgical ablation, an extensive valve annuloplasty was often performed to ensure AP interruption. ${ }^{19}$ These patients with diffuse connections in our study population, therefore, may have had less optimal ablation results than those with more discrete connections. Our present study also shows that patients with true recurrence are typically younger at the time of ablation. The younger age of the patient and nonlocalized distribution of the fibers as described above may preclude more aggressive ablation in order to reduce damage to the normal conduction system. Therefore, this may account for a higher recurrence rate in our study.

ST is common $(30-60 \%)$ in the postablation period. $^{4,8}$ All episodes of ST, however, do not indicate true SVT recurrence and may only represent heightened sensitivity to sinus tachycardia. Among the few published studies, Mann et al. interviewed 77 patients, 4 weeks after undergoing ablation for SVT. ${ }^{8}$ Of these, $58 \%(\mathrm{~N}=45)$ reported ST but only $24 \%(8 / 32)$ were found to have evidence of recurrent AP on repeat EP study done after 3 months. Tomaske et al. followed 97 pediatric patients for 6 months after ablation. Of these, only $6 \%$ had true recurrence. ${ }^{4}$ The literature already has multiple "acute" success studies and limited 4-6 months follow-up results. Nevertheless, distinguishing true episodes of recurrence from sensed sinus tachycardia remains a common diagnostic challenge for the clinician. While several tests are routinely employed to identify "true" episodes, our study is one of the first to look at the diagnostic utility of these tests. Recently, AEMs have gained popularity in the evaluation of palpitations in children.,10 Park et al. studied 30 children who underwent AEM for symptoms of palpitations and were accurately diagnosed. ${ }^{11}$ In a previous study at our institution, 202 symptomatic children were evaluated with either 24-hour Holter monitor $(\mathrm{N}=$ 97) or telephone AEMs $(N=105)$. The study showed that 30-day AEMs had a better correlation of symptoms with arrhythmias $(32 \%$ vs $5 \%$ for Holter). ${ }^{9}$ Our present study also shows that AEMs had the highest sensitivity and specificity in identifying true SVT recurrence. However, up to $29 \%$ of events may still be missed with AEMs in symptomatic patients. The possible reasons could be patient noncompliance in following instructions or difficulty in transmitting the data. Newer devices, with patient-friendly capabilities may have better sensitivity in detecting these episodes and further studies are required to evaluate their diagnostic utility.

The study has some limitations. This was a retrospective study from a single institution. Complaints of ST were evaluated without a standardized protocol and based on clinical judgment and therefore the results of testing were nonuniform. Also the recurrence rates in patients who were lost to follow-up cannot be estimated. However, despite these limitations, the study provides useful clinical information about the long-term outcomes of ablation therapy of SVT in children. Our large study sample and long followup period allows for realistic estimation of acute and long-term success rates in these patients. The study also focuses on a very common clinical problem of ST and hopes to provide some insight into the utility of diagnostic testing.

\section{Conclusions}

Acute success following ablation in the EP lab does not define chronic ablation success. Subjective complaints of ST are common in children; however, true recurrences occurred in only one-fourth of patients and can be related to the location of the AP. Although event monitors are the most sensitive diagnostic tool for predicting true recurrence, the diagnosis can still be missed in a significant number of patients. Longitudinal studies comparing different types of event monitors are needed for most cost-effective management of this common clinical problem. Persistent clinical monitoring of children following ablation is warranted.

\section{References}

1. Ko JK, Deal BJ, Strasburger JF, Benson DW Jr. Supraventricular tachycardia mechanisms and their age distribution in pediatric patients. Am J Cardiol 1992; 69:1028-1032.

2. Ludimorsky A, Garson A Jr. Supraventricular tachycardia. In: Pediatric Arrhythmias: Electrophysiology and Pacing. Philadelphia, WB Saunders, 1990, p. 380.

3. LaPage MJ, Saul JP, Reed JH. Long-term outcomes for cryoablation of pediatric patients with atrioventricular nodal reentrant tachycardia. Am J Cardiol 2010; 105:1118-1121.

4. Tomaske M, Candinas R, Weiss M, Bauersfeld U. Safety and efficacy of paediatric outpatient radiofrequency catheter ablations. Int J Cardiol 2009; 148:276-279. 
5. Buddhe S, Singh H, Du W, Karpawich PP. Radiofrequency and cryoablation therapies for supraventricular arrhythmias in the young: Five-year review of efficacies. Pacing Clin Electrophysiol 2012; 35:711-717

6. Van Hare GF, Javitz H, Carmelli D, Saul JP, Tanel RE, Fischbach PS, Kanter RJ, et al. Prospective assessment after pediatric cardiac ablation: Demographics, medical profiles, and initial outcomes. J Cardiovasc Electrophysiol 2004; 15:759-770.

7. Riggs TW, Byrd JA, Weinhouse E. Recurrence risk of supraventricular tachycardia in pediatric patients. Cardiology 1999; 91 : 25-30.

8. Mann DE, Kelly PA, Adler SW, Fuenzalida CE, Reiter MJ. Palpitations occur frequently following radiofrequency catheter ablation for supraventricular tachycardia, but do not predict pathway recurrence. Pacing Clin Electrophysiol 1993; 16:16451649.

9. Karpawich PP, Cavitt DL, Sugalski JS. Ambulatory arrhythmia screening in symptomatic children and young adults: Comparative effectiveness of Holter and telephone event recordings. Pediatr Cardiol 1993; 14:147-150.

10. Saarel EV, Doratotaj S, Sterba R. Initial experience with novel mobile cardiac outpatient telemetry for children and adolescents with suspected arrhythmia. Congenit Heart Dis 2008; 3: 33-38.

11. Park MH, de Asmundis C, Chierchia GB, Sarkozy A, Benatar A, Brugada P. First experience of monitoring with cardiac event recorder electrocardiography Omron system in childhood population for sporadic, potentially arrhythmia-related symptoms. Europace 2011; 13:1335-1339.
12. Draper DE, Giddins NG, McCort J, Gross GJ. Diagnostic usefulness of graded exercise testing in pediatric supraventricular tachycardia. Can J Cardiol 2009; 25:407-410.

13. Spar DS, Silver ES, Hordof AJ, Liberman L. Relation of the utility of exercise testing for risk assessment in pediatric patients with ventricular preexcitation to pathway location. Am J Cardiol 2011; 109:1011-1014.

14. Chetaille P, Walsh EP, Triedman JK. Outcomes of radiofrequency catheter ablation of atrioventricular reciprocating tachycardia in patients with congenital heart disease. Heart Rhythm 2004; 1:168173.

15. Triedman JK, Alexander ME, Love BA, Collins KK, Berul CI, Bevilacqua LM, Walsh EP. Influence of patient factors and ablative technologies on outcomes of radiofrequency ablation of intra-atrial re-entrant tachycardia in patients with congenital heart disease. J Am Coll Cardiol 2002; 39:1827-1835.

16. Harahsheh A, Du W, Singh H, Karpawich PP. Risk factors for atrioventricular tachycardia degenerating to atrial flutter/fibrillation in the young with Wolff-Parkinson-White. Pacing Clin Electrophysiol 2008; 31:1307-1312.

17. Ho S. Accessory atrioventricular pathways: Getting to the origins. Circulation 2008; 117:1502-1504.

18. Iturralde P, Guevara-Valdivia M, Rodriquez-Chavez L, Medeiros A, Colin L. Radiofrequency ablation of multiple accessory pathways. Europace 2002; 4:273-280.

19. Misaki T, Watanabe G, Iwa T, Matsunaga Y, Ohotake H, Tsubota M, Takahashi M, et al. Surgical treatment of patients with WolffParkinson-White syndrome and associated acquired valvular heart disease. J Thorac Cardiovasc Surg 1994; 108:68-72. 